\title{
Interventional Vascular Surgery Guidance during the COVID-19 Pandemic
}

\author{
Amr Abdelghaffar Mahmoud MD \\ Vascular Surgery Department, Ain Shams University, Cairo, Egypt
}

\begin{abstract}
Background: The ongoing outbreak of the COVID-19 pandemic is considered a major public concern and has resulted in a huge burden on health care. We hereby provide a brief review for vascular surgeons regarding COVID-19 clinical issues, pathogenesis, diagnosis, and treatment approaches.

Methods: We review the international society's guidance on managing vascular interventions during the COVID-19 outbreak.

Results: This work provides summarized descriptions of how to manage and deal with vascular surgery cases at time of COVID-19 outbreak, including measures at outpatient clinic, emergency room, and operations.

Conclusions: Although vascular surgeons are not considered to be the front line in combating the COVID-19 pandemic, certain measures, detailed in this work, can be adopted in their practice during this period to control and lessen the spread of the disease.
\end{abstract}

Keywords: COVID-19; Surgery During Pandemic; COVID-19 and Vascular Practice

\section{INTRODUCTION}

The ongoing outbreak of COVID-19, coronavirus disease 2019 , is considered a major public concern. It was first identified in December 2019 in Wuhan, China, and has spread to most countries of the world. The World Health Organization (WHO) declared it a pandemic on the 11 March 2020 and called on countries to take urgent actions to control its spread [1]. The information in this review is updated to June 2020. Briefly, Coronaviruses are enveloped, positive single-stranded large RNA viruses that infect humans but also a wide range of animals [2]. The COVID-19 virus shares $96 \%$ of its whole-genome with a bat coronavirus causing the accusation that bats were the origin of the virus [4]. Disease transmission

\section{Corresponding author:}

Amr Abdelghaffar Mahmoud, MD, lecturer and consultant at vascular surgery department, Ain Shams University, 56 Ramsees Street, Abbasia, Cairo, Egypt.

Email: amr.mahmoud@med.asu.edu.eg

(C) 2020 CC BY 4.0 - in cooperation with Depts. of Cardiothoracic/ Vascular Surgery, General Surgery and Anesthesia, Örebro University Hospital and Örebro University, Sweden mainly occurs via human respiratory droplets and direct contact from symptomatic or even asymptomatic carriers with a mean incubation period of 5 days $[5,6]$. At present, there are no specific antiviral drugs or vaccines against the COVID-19 infection for potential therapy in humans and the current treatment is mainly supportive. The elderly, the immunocompromised, those with ischemic heart disease or diabetes, men, people with high Body Mass Index (BMI), hypertension and smokers appear to be at a greater risk of severe complications when infected and these demographics are highly pertinent to vascular patients. Although vascular surgeons are not considered as the frontline in combating the pandemic of COVID-19, certain measures can be adopted in their practice during this period to control and lessen the spread of the disease. These measurements are based on rapid guidelines adopted by international societies and summarized in this article.

\section{METHODS}

A general review of current guidelines of major vascular and surgical societies was carried out and updated to April 2020. Pubmed and surgical society web sites where used for data search. 


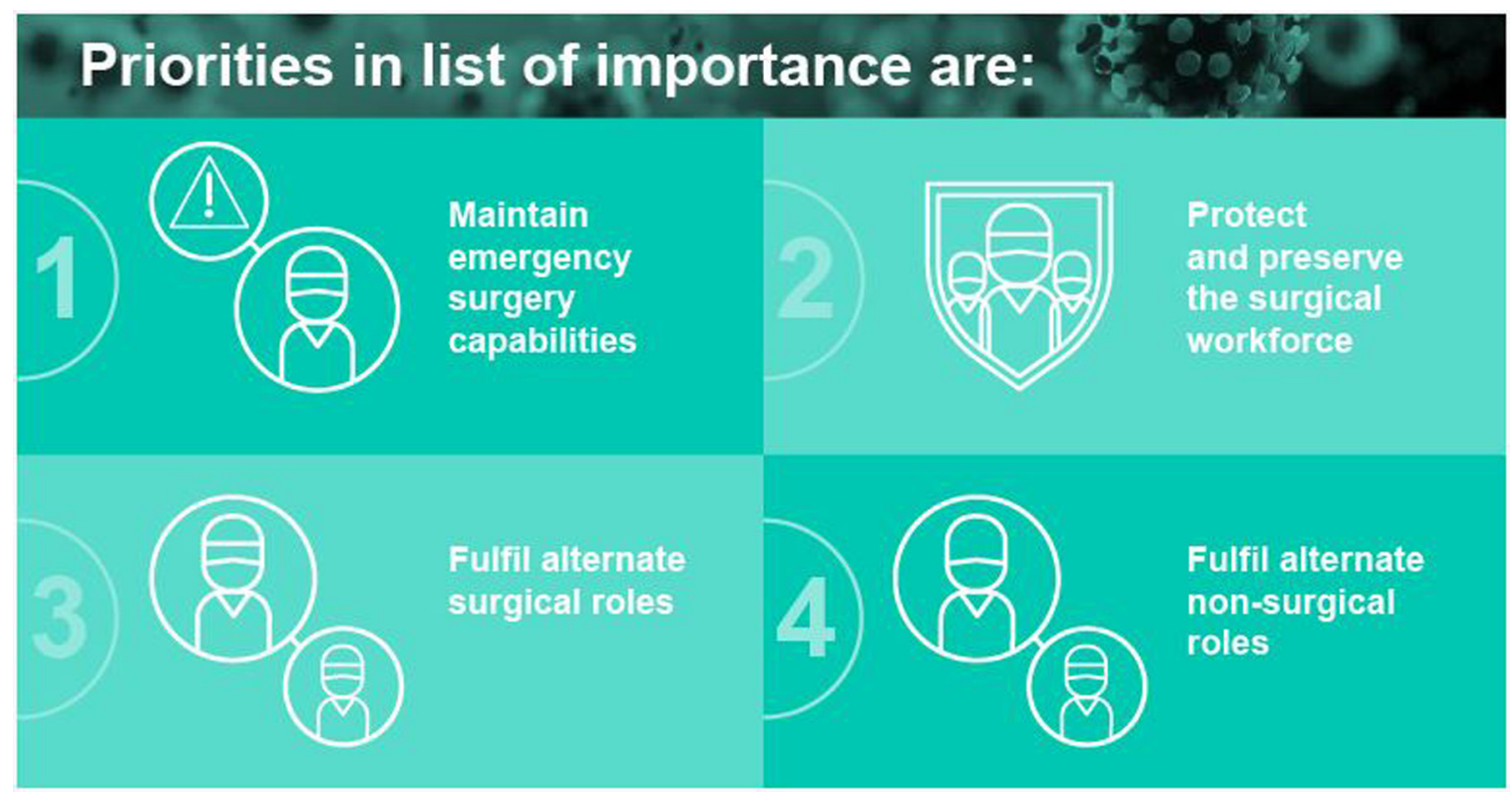

Figure 1 Priorities listed by importance [16].

\section{Summary of Vascular Surgery Measures and discussion}

The advice given by the Vascular Society of Great Britain and Ireland is that, where possible, only urgent outpatients should be seen, and virtual clinics should be considered [12]. The American College of Surgeons recommends that all non-urgent in-person clinic/office visits should be canceled or postponed, unless needed to triage active symptoms or manage wound care. It is recommended that elective urgent inpatient diagnostic and surgical procedures are shifted to outpatient settings when feasible [13]. The Vascular Society of Great Britain and Ireland also suggests that surgeons in training will have key roles to play in this crisis but the underlying principles of appropriate supervision, working practices, rest, and pastoral care remain [12].

\section{Clinical interpretation of the guidelines (Figure 1)}

Maintenance of emergency surgery capabilities is achieved as follows:

1. Maintaining Emergency surgery provision, including major trauma (MT), must be achieved for the surgery workforce.

2. Initially, this will be delivered by individual specialty rotas. These will include rotas where some members of the team do not come into work and act as a healthy reserve.

3. If the workforce is reduced and it may be necessary to move to a generic surgeon rota, based around competencies and would manage the initial triage, some of the surgery, and the post-operative management.
4. It is likely that the workforce will break down into torso/cavity surgeons (vascular, general, urology) and extremity surgeons (orthopedics, plastics). Virtual support from specialists would be required for the generic surgeon. Some of the operations will still require a specialist surgeon when available.

5. It is suggested that each site would have a torso surgeon and an extremity surgeon with middle grades present. The shift pattern would be $24 \mathrm{~h}$ or $12 \mathrm{~h}$ depending on numbers of surgeons and tempo.

6. The MT pathway has been identified as a national priority. Surgeons may be required to take over running of the MT service, including trauma team leader (TTL) role, depending on local arrangements.

7. Regional solutions may be required if smaller surgical units collapse. The existing MT networks are likely to be the best vehicle to achieve this [18].

Due to the pressure on emergency departments, non-respiratory emergencies may be triaged to an alternate pathway which may need support from surgeons (Non-COVID Emergency Department). This could be incorporated into providing TTL cover for MT centers/ trauma units, depending on existing local arrangements [16].

Fulfillment of alternate surgical roles is achieved as follows:

1. If all other priorities have been met and the surgical workforce has been maintained, it may be possible for some surgeons to take on non-surgical roles.

2. These could include running level $2 / 3$ units or non-clinical roles in command and control. Individuals 


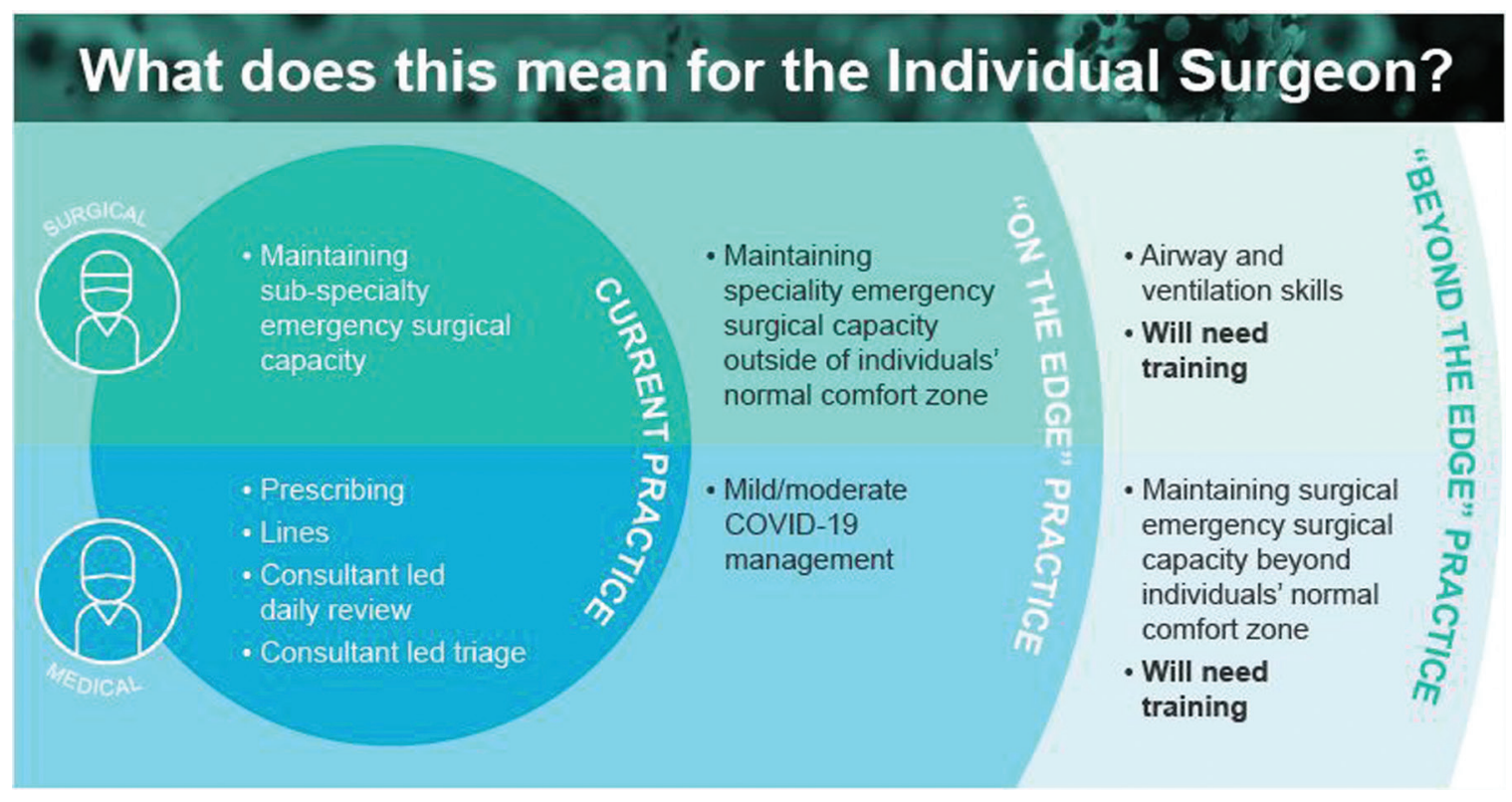

Figure 2 What does this mean for the individual surgeon [16]?

will need training/mentoring/support in these roles outside their normal practice [18].

To explain what this means for each individual surgeon, there are 3 zones of practice as follows:

(A) Current practice. Medical - prescribing, lines, consultant led daily review, and consultant led triage. Surgical - maintaining sub-specialty emergency surgical capacity.

(B) "On the edge" practice. Medical - mild/moderate COVID-19 management. Surgical - maintaining specialty emergency surgical capacity outside of individuals' normal comfort zone.

(C) "Beyond the edge" practice. Medical - airway and ventilation skills will need training. Surgical - Maintaining emergency capacity beyond individuals' normal comfort zones. (Figure 2) [16].

The American College of Surgeons provide the "By the Well-Being Index" for health care administrators to reduce stress and pressure upon Health Care Workers (HCWs). Applying some or all of them will substantially increase the overall well-being of staff; living accommodation should be offered for intensive care unit (ICU)/ Emergency Room (ER) physicians who live with a vulnerable family member, so that they do not have to go home (e.g. on-campus, hotel). Meal credits should be provided (e.g. Uber eats, delivery) for those working extra shifts or unanticipated overtime. Dictation and transcription services should be made available to all in the ICU/hospital/ER. HCWs with known health conditions, which place them at an elevated risk for complications should they contract COVID-19, should be redeployed to other settings. Workers should be required to take breaks to recharge and adequate time off between shifts should be encouraged. All required online modules and non-essential tasks should be halted. Taxi and ridesharing fare reimbursement should be provided to all employees directly engaged in COVID-19 efforts [13].

\section{Institutional and specific area of treatment guidelines}

In Ain Shams University, Egypt, the number of trainees per shift has been reduced by half, the number of intern doctors has also been reduced by half, the number of operations has been reduced, and non-emergent or urgent cases have been postponed. The decision to intervene or postpone is made by the head of each surgical department at weekly scientific meetings. All educational platforms and lectures have cancelled and replaced by meetings over the internet. Triage areas in ERs have been established to isolate feverish and suspected cases to avoid mixing with non-suspected cases. All HCWs in ERs use PPE in the form of gowns, masks, and an eye shield, while HCWs in operation rooms only wear masks in between operations. We also advise staying home and self-monitoring for those who have come into contact with suspected cases, until COVID-19 results are collected. Positive COVID-19 of HCWs are isolated at a quarantine-hospital outside Cairo. This belongs to Ain Shams University Hospitals, which has ICU beds and mechanical ventilators. The Center for Disease Control and Prevention provides a suggested method for using PPE (Figures 3 and 4) [17].

\section{On-Call Arrangements}

The Vascular Society of Great Britain and Ireland advises that a second on-call consultant is available to help with 


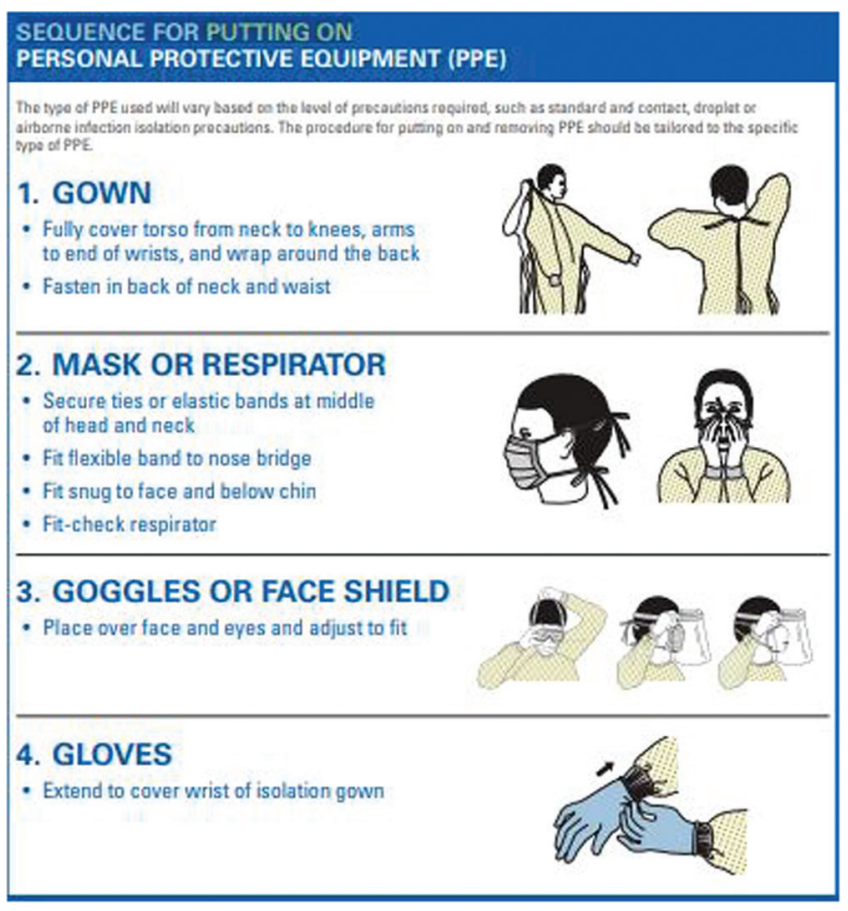

Figure 3 Putting on PPE [17].

the emergency workload (and also if self-isolation becomes common) [12]. A vascular consultant surgeon should be on call and available to see all referrals. Trusts should consider having another vascular surgeon on call for performing surgery [12].

\section{Elective Surgery}

The Vascular Society of Great Britain and Ireland advises that elective arterial surgery and venous surgery should be deferred. Asymptomatic carotid surgery and surgery for claudication should be deferred [12].

\section{Aortic Surgery}

The Vascular Society of Great Britain and Ireland advises that the size threshold for AAA surgery needs to weigh up the risk of rupture in the next few months against the risk from intervention and resource limitation [12]. It advises that ruptured aneurysms should ideally be treated by endovascular aortic repair (EVAR) whenever possible to reduce dependence on the High Dependency Unit and reduce the length of stay. Open surgery should only be considered when EVAR is inappropriate or unavailable and in cases where there is a good chance of success. ITU capacity will need to be considered prior to intervention [12].

The American College of Surgeons has recently published COVID-19 guidelines for the triage of vascular surgery patients including aortic diseases (Table 1) [13].

\section{Acute Ischemia/Critical Leg Ischemia \pm Foot Infection}

The Vascular Society of Great Britain and Ireland advises that there may be situations where primary amputation may be more appropriate than complex revascularizations, multiple debridements, and potential prolonged hospital stay [12].

A pathway for guidance has been developed by a collaborative group of expert clinicians in Foot in Diabetes UK (FDUK) to support all lower-limb clinicians during the COVID-19 situation in line with current best practice. It includes the assessment of the following conditions:

(1) Non limb-threatening problems: leg or foot pain that is not due to severe infection or ischemia, superficial leg/foot ulcers that show evidence of healing, asymptomatic peripheral arterial disease or intermittent claudication only, foot pulses non-palpable or monophasic on Doppler (asymptomatic), mild foot or leg infections with shallow ulcers and local erythema $<2 \mathrm{~cm}$ from the edge with no signs of tracking or sepsis, and acute Charcot feet without infection (to be completely rested/offloaded). All the previous cases should be treated, monitored, or advised by an appropriately skilled lower limb clinician or general practitioner, using local infection wound care and pain management guidelines or protocols where available.

If previous conditions deteriorate and develop key indications of limb-threating infection or sepsis or critical limb ischemia, consultation and intervention is required [20].

(2) Limb-threatening infection or sepsis: deteriorating/ tracking infection, especially with ulcer depth to bone or critical limb ischemia, spreading cellulitis in the foot or leg (e.g. redness, swelling, pus, heat, pain, black discoloration) without sepsis, or with sepsis indicated by pulse rate $<50$ beats per minute (BPM) or $>90$ BPM, respiration rate $<11$ or $>20$ breaths per minute, flu-like symptoms, and being confused/unresponsive/drowsy (these features could also be caused by COVID-19 infection). All previous cases require consultation and intervention [18].

(3) Critical limb ischemia: foot pulses not palpable/ absent, Doppler signals monophasic/absent, indications of Buerger's disease (foot goes pale on elevation and goes red when hung down), ankle systolic blood pressure $<50 \mathrm{mmHg}$, and toe systolic blood pressure $<30 \mathrm{mmHg}$. In addition to these are any of the following: ischemic rest pain in toes/feet for more than 2 weeks, new gangrene or necrosis, acute limb ischemia, and sudden onset cold, pale, pulseless, painful limb, especially if also developing paresthesia or paralysis. All previous conditions require consultation and intervention [18].

Consultation and intervention are in the form of urgent discussions with high-risk foot podiatry, hospital vascular, diabetes foot, infectious disease, or orthopedic 


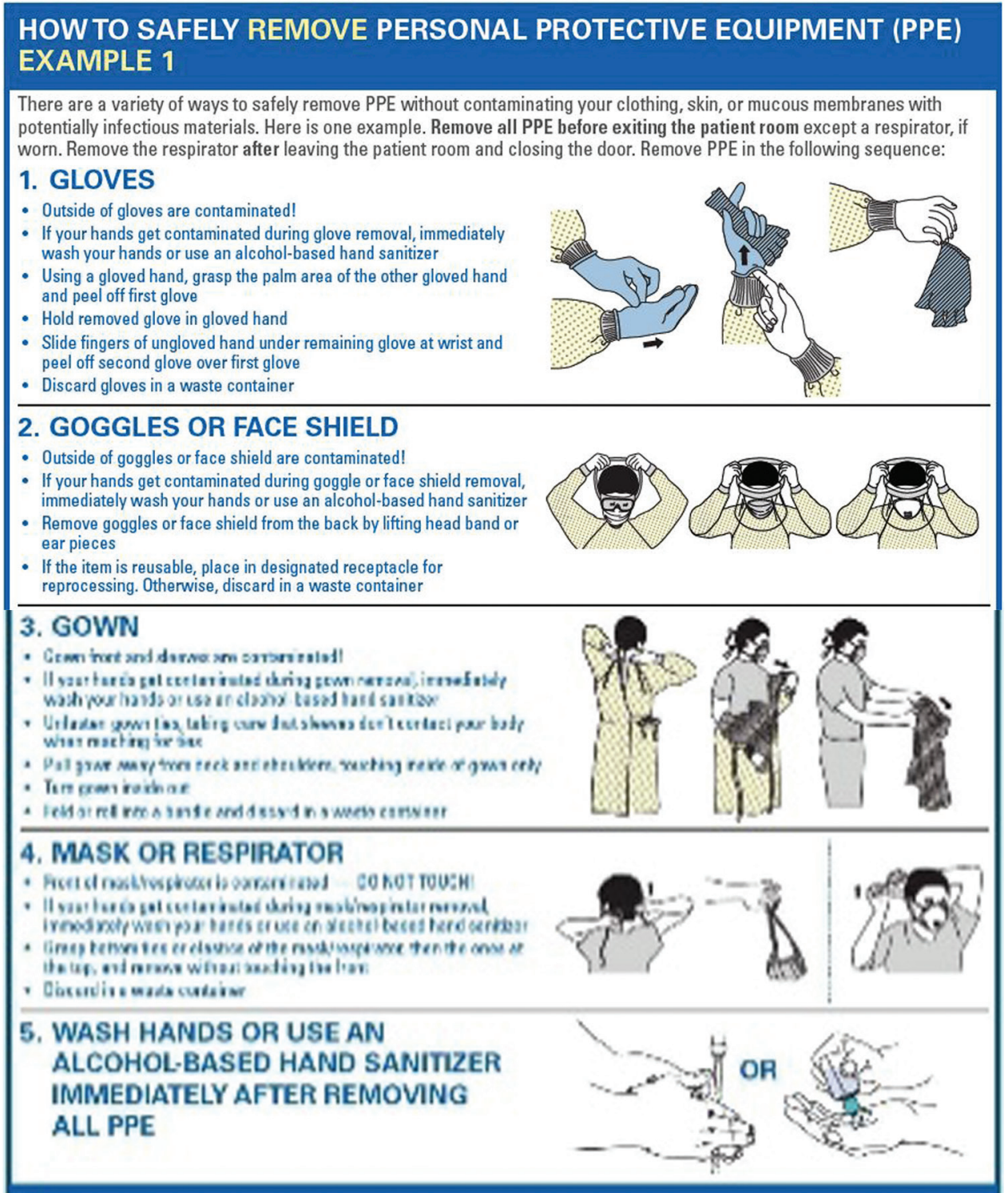

Figure 4 Removal of PPE [17].

Table 1 American College of Surgeons' COVID-19 guidelines for triage of vascular surgery patients and aortic diseases [21].

\begin{tabular}{lll}
\hline Category & Condition & Tier Class \\
\hline AAA & Ruptured or symptomatic TAAA or AAA & 3. Do not postpone \\
& Aneurysm associated with infection or prosthetic graft infection & 3. Do not postpone \\
& AAA $>6.5 \mathrm{~cm}$ & 2b. Postpone if possible \\
& TAAA $>6.5 \mathrm{~cm}$ & 2b. Postpone if possible \\
& AAA $<6.5 \mathrm{~cm}$ & 1. Postpone \\
Aortic dissection & Acute aortic dissection with rupture or malperfusion & 3. Do not postpone \\
Aortic emergency NOS & AEF with septic/hemorrhagic shock, or signs of impending rupture & 3. Do not postpone \\
\hline AAA, abdominal aortic aneurysm; TAAA, Thoracic aortic aneurysm; AEF, Aorto-enteric fistula; NOS, not otherwise specified
\end{tabular}

multi-disciplinary teams. The vascular team or the on-call vascular/surgical registrar must be contacted immediately for discussions. If the clinical situation appears acute and life- or limb-threatening, intervention may be needed. The team must be informed of the COVID-19 infection status, if known [18].

The American College of Surgeons recently published COVID-19 guidelines for triage of vascular 
Table 2 American College of Surgeons' COVID-19 guidelines for triage of vascular surgery patients including peripheral vascular diseases [21].

\begin{tabular}{|c|c|c|}
\hline Category & Condition & Tier Class \\
\hline \multirow[t]{5}{*}{ Aneurysm peripheral } & Peripheral aneurysm, symptomatic & 3. Do not postpone \\
\hline & Peripheral aneurysm, asymptomatic & 2a. Consider postponing \\
\hline & $\begin{array}{l}\text { Pseudoaneurysm repair: not a candidate for } \\
\text { thrombin injection or compression, rapidly } \\
\text { expanding, complex }\end{array}$ & 3. Do not postpone \\
\hline & $\begin{array}{l}\text { Symptomatic non-aortic intra-abdominal } \\
\text { aneurysm }\end{array}$ & 3. Do not postpone \\
\hline & $\begin{array}{l}\text { Asymptomatic non-aortic intra-abdominal } \\
\text { aneurysm }\end{array}$ & 2a. Consider postponing \\
\hline \multirow[t]{3}{*}{ Bypass graft complications } & $\begin{array}{l}\text { Infected arterial prosthesis without overt sepsis } \\
\text { hemorrhagic shock, or impending rupture }\end{array}$ & 3. Do not postpone \\
\hline & $\begin{array}{l}\text { Revascularization for high grade re-stenosis of } \\
\text { previous intervention }\end{array}$ & 2b. Postpone if possible \\
\hline & Asymptomatic bypass graft/stent restenosis & 1. Postpone \\
\hline \multirow[t]{6}{*}{ Peripheral vascular disease } & Acute limb ischemia & 3. Do not postpone \\
\hline & $\begin{array}{l}\text { Limb ischemia: progressive tissue loss, acute } \\
\text { limb ischemia, wet gangrene, ascending } \\
\text { cellulitis }\end{array}$ & 3. Do not postpone \\
\hline & Fasciotomy for compartment syndrome & 3. Do not postpone \\
\hline & $\begin{array}{l}\text { Peripheral vascular disease: chronic limb-threat- } \\
\text { ening ischemia, rest pain, or tissue loss }\end{array}$ & 2b. Postpone if possible \\
\hline & $\begin{array}{l}\text { Peripheral angiograms and endovascular } \\
\text { therapy for claudication }\end{array}$ & 1. Postpone \\
\hline & Surgical procedures for claudication & 1. Postpone \\
\hline \multirow[t]{5}{*}{ Wounds/Gangrene/Amputation } & $\begin{array}{l}\text { Amputations for infection/necrosis (TMA, BKA, } \\
\text { AKA) }\end{array}$ & 3. Do not postpone \\
\hline & $\begin{array}{l}\text { Lower extremity disease with non-salvageable } \\
\text { limb (amputation) }\end{array}$ & 3. Do not postpone \\
\hline & $\begin{array}{l}\text { Deep debridement of surgical wound infection } \\
\text { or necrosis }\end{array}$ & 2b. Postpone if possible \\
\hline & Wounds requiring skin grafts & 2b. Postpone if possible \\
\hline & Amputations for infection/necrosis (toes) & 2b. Postpone if possible \\
\hline
\end{tabular}

TMA, Trans-metatarsal amputation; BKA, Below knee amputation; AKA, Above knee amputation

surgery patients including peripheral vascular diseases (Table 2) [21].

\section{Diabetic Foot and Podiatric}

Podiatric care is associated with fewer diabetes-related amputations, ER visits, hospitalizations, lower length-ofstay, and lower costs. However, podiatrists must mobilize and adopt the new paradigm of shifts away from hospital care to community-based care. Implementing the proposed Pandemic Diabetic Foot Triage System in-home visits, higher acuity office visits, telemedicine, and remote patient monitoring can help podiatrists manage patients while reducing the COVID-19 risk. The goal of podiatrists during the pandemic is to reduce the burden on the health care system by keeping diabetic foot and wound patients safe, functional, and at home [21]. Podiatrists have adapted quickly to the new pandemic system of care and made changes so as to provide services in new and unique ways. They strongly recommend implementing a triage system for lower-extremity wounds and diabetic foot problems, which will drive the site and urgency of podiatric care (Figure 5) [19].

\section{Carotid Surgery}

The Vascular Society of Great Britain and Ireland advises that crescendo Transient Ischemic Attacks (TIAs) would normally need urgent surgery. If there are severe resource limitations, aggressive pursuit of the 


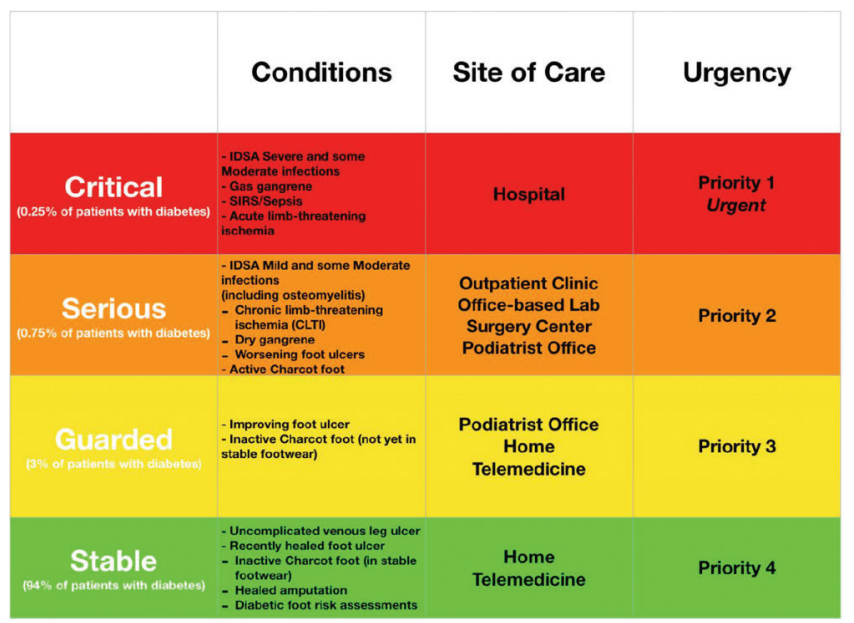

Figure 5 Priority and urgency for podiatric conditions.

best medical therapy would be more appropriate for recently symptomatic carotid disease [12].

The American College of Surgeons' COVID-19 guidelines for triage of vascular surgery patients advise not to postpone symptomatic carotid stenosis, while it advises to postpone asymptomatic ones [21].

\section{Venous Surgery}

The Vascular Society of Great Britain and Ireland advises that elective arterial surgery and venous surgery should be deferred [12].

The American College of Surgeons' COVID-19 guidelines for triage of vascular surgery patients advise the postponement of asymptomatic patients with MayTurner syndrome, patients with varicose veins, and Inferior Vena Cava (IVC) filter removal. It advises consideration of the postponement for massive symptomatic ilio-femoral deep venous thrombosis (DVT) in low risk patients, procedures for ulcerations secondary to venous disease, and IVC filter placement [21]. It also advises proceeding with intervention in cases of acute ilio-femoral DVT with phlegmasia [21].

For trauma, it advises proceeding with intervention in cases of traumatic injury with hemorrhage and/or ischemia or surgery/embolization for uncontrolled bleeding in unstable patients. Otherwise it advises postponement if possible in cases of surgery/embolization for bleeding in stable patients [21].

For mesenteric vascular disease, it advises proceeding with intervention and no postponement for symptomatic acute mesenteric occlusive disease. However, it advises considering postponement for cases of chronic mesenteric ischemia [21].

For thoracic outlet syndrome (TOS), it advises postponement for cases of neurogenic and mild venous TOS and postponement, if possible, for cases of arterial thoracic outlet syndrome with thrombosis or symptomatic venous TOS with acute occlusion and marked swelling [21].

\section{Renal Dialysis}

Rapid National Institute for Health and Care Excellence (NICE) recommendations for dialysis service delivery include the following:

(1) Communicating with patients: communicate with patients and support their mental wellbeing to help alleviate any anxiety and fear. Tell patients to alert their dialysis unit if they are unwell. Minimize faceto-face contact by offering telephone or video consultations, cutting non-essential face-to-face follow-up, using home-delivery services for medicines, and using local services for blood tests [20].

(2) Patients not known to have COVID-19: encourage patients to use their own transport and to travel alone to the dialysis unit when possible. Minimize time in the waiting area by careful scheduling, encouraging patients not to arrive early, and texting patients when the unit is ready to see them [20].

(3) Patients known or suspected to have COVID-19: follow the national guidance on infection prevention and control.

(4) Patient transport to and from dialysis units: ensure that outpatient transport services get patients to their dialysis as scheduled to avoid their condition deteriorating. Work with transport providers to have arrangements in place to ensure continuity in patient care. Collaborate with the transport provider to minimize cross-infection between patients with known COVID-19 and those suspected of having COVID-19 [20].

(5) Before patients enter the unit for dialysis: screen and triage patients before they enter the dialysis unit (for example, at the reception waiting area). If people are suspected of having COVID-19, where possible, carry out rapid turnaround testing before dialysis to establish COVID-19 status. Dialysis may be needed before the test results are available [20].

(6) Home dialysis provision: Continue and maintain current home dialysis provision (home hemodialysis and peritoneal dialysis) and maintain adequate supplies and staffing support. Test for COVID-19 in patients, carers, and assistants (paid and unpaid) in the community using any form of home dialysis if they develop symptoms. Test paid assistants carrying out assisted automated peritoneal dialysis [20].

From another point of view, the American College of Surgeons advise proceeding with intervention and not postponing cases of thrombosed or nonfunctional dialysis access, infected dialysis access, fistula revision for ulceration, renal failure with need for dialysis access, and tunneled dialysis catheter. They advise postponing, 
if possible, cases of fistula revision for malfunction, vascular access steal syndrome and fistulagram for vascular access malfunction. They advise considering postponement for cases of Arterio-venous (AV) fistula and graft placement for dialysis (End Stage Renal Disease (ESRD), chronic kidney disease stage 4, chronic kidney disease stage 5 only) [21].

We suggest that vascular access operations are lifeline operations; they should be continued especially when they are based on "single-day operations" with no need for admission. Patients diagnosed with venous hypertension low-grade steal syndrome should be managed conservatively. Patients diagnosed with chronic kidney disease and who have not started dialysis yet should be postponed.

\section{Surgical Measures at Ain Shams University Hospital}

(1) Specialized hospital treatment for all patients as if infected with COVID-19 until proven otherwise and requests for laboratory testing before intervention except in emergent cases.

(2) The general hospital (limited resources) provides a surgical service for non-COVID-19 patients aimed at the clinical picture (no fever, cough, leucopenia), while suspected clinical or confirmed cases are transferred to Obour hospital (university quarantine hospital) outside of Cairo which provides surgical and medical care.

(3) There are a limited number of operation lists and limited times of operations. The vascular surgery operation list has been reduced from five to three times per week.

(4) Surgical intervention is limited to urgent and emergent cases based on a weekly meeting consultation by the head of department and other consultants.

(5) Cleaning of the operation room is intensively carried out under direct supervision of the operation room manager and head nurse.

(6) Regular cleaning and disinfection of surfaces by sodium-hypochlorite disinfectant is carried out and hand alcohol-based disinfectants are available everywhere.

(7) Well-spaced feet signs have been put in elevators, with no face-to-face communication in close and limited spaces.

(8) Patients have no time to wait in the induction room; they arrive from the ward and immediately enter the operation room. The recovery room has separate sectors for isolation and only half the number of rooms are on demand so there are fewer patients in the recovery room.

(9) Admissions are served for non COVID-19 suspected emergent or urgent cases only, and visits by patient's relatives are limited. There are enough spaces between patients, with good ventilation.
(10) HCWs use personal protective equipment (PPE) in the emergency triage area, such as gowns, surgical masks, and eye shields, while they use only masks in the operation rooms and ward. Alcohol-based antiseptic solutions are available everywhere.

\section{CONCLUSIONS}

Although vascular surgeons are not considered to be the front line in combating the pandemic of COVID-19, certain measures can be adopted in their practice during this period to control and lessen the spread of the disease.

\section{Ethics Statement}

(1) All the authors mentioned in the manuscript have agreed to authorship, read and approved the manuscript, and given consent for submission and subsequent publication of the manuscript.

(2) The authors declare that they have read and abided by the JEVTM statement of ethical standards including rules of informed consent and ethical committee approval as stated in the article.

\section{Conflicts of Interest}

The author declares that they have no conflicts of interest.

\section{Funding}

The author received no financial support for the research, authorship, and/or publication of this article.

\section{REFERENCES}

[1] World Health Organization. Situation reports. https:// www.who.int/dg/speeches/detail/who-director-generals-opening-remarks-at-the-media-briefing-on-covid-1911-march-2020. Accessed 22 March 2020.

[2] Tyrrell DA, Bynoe ML. Cultivation of viruses from a high proportion of patients with colds. Lancet. 1966;1: 76-7.

[3] GISAID Global Initiative on Sharing All Influenza Data. Phylogeny of SARS-like betacoronaviruses including novel coronavirus (nCoV). https://nextstrain.org/groups/ blab/sars-like-cov. Accessed 26 February 2020.

[4] Zhou P, Yang XL, Wang XG, et al. A pneumonia outbreak associated with a new coronavirus of probable bat origin. Nature. 2020; In Press. doi: 10.1038/s41586-0202012-7.

[5] Rothe C, Schunk M, Sothmann P, et al. Transmission of 2019-nCoV infection from an asymptomatic contact in Germany. N Engl J Med. 2020;382:970-1

[6] Guan W, Ni Z, Yu H, et al. Clinical characteristics of 2019 novel coronavirus infection in China. N Engl J Med. In Press. doi: 10.1056/NEJMoa2002032.

[7] Holshue ML, DeBolt C, Lindquist S, et al. First case of 2019 novel coronavirus in the United States. N Engl J Med 2020;382:929-36. 
[8] Lu R, Zhao X, Li J, et al. Genomic characterisation and epidemiology of 2019 novel coronavirus: implications for virus origins and receptor binding. Lancet. 2020. In Press. doi: 10.1016/S0140-6736(20)30251-8.

[9] Kampf G, Todt D, Pfaender S, Steinmann E. Persistence of coronaviruses on inanimate surfaces and its inactivation with biocidal agents. J Hosp Infect. 2020;104:246-51.

[10] Huang C, Wang Y, Li X, Ren L, et al. Clinical features of patients infected with 2019 novel coronavirus in Wuhan, China. Lancet. 2020;395(10223):497-506.

[11] H. Lu. Drug treatment options for the 2019-new coronavirus (2019-nCoV). Biosci Trends. 2020;14(1):69-71.

[12] Vascular Society of Great Britain and Ireland. COVID-19 virus and vascular surgery. https://www.vascularsociety. org.uk/professionals/news/113/covid19_virus_and_ vascular_surgery. Accessed 20 March 2020.

[13] American College of Surgeons. COVID-19: recommendations for management of elective surgical procedures. https://www.facs.org/about-acs/covid-19/information-for-surgeons/elective-surgery. Accessed 13 March 2020.

[14] Liang ZC, Wang W, Murphy D, Po Hui JH. Novel Coronavirus and orthopaedic surgery: early experiences from Singapore. J Bone Joint Surg Am. 2020. In Press. doi: 10.2106/JBJS.20.00236.

[15] Society of American Gastrointestinal and Endoscopic Surgeons (SAGES). SAGES and EAES Recommendations regarding surgical response to COVID-19 crisis. https:// www.sages.org/recommendations-surgical-responsecovid-19/. Accessed 19 March 2020.

[16] Royal College of Surgeons. Guidance for surgeons working during the COVID-19 pandemic https://www.rcseng. ac.uk/coronavirus/joint-guidance-for-surgeons-v1/. Accessed 20 March 2020.

[17] Centers for Disease Control and Prevention. PPE sequence. https://www.cdc.gov/hai/pdfs/ppe/ppe-sequence.pdf. Accessed May 2018.

[18] Foot in Diabetes UK. COVID-19 situation v1.3 lower limb amputation prevention guidance. https://www.wounds-uk. com/resources/details/lower-limb-amputation-prevention-guidance. Accessed 23 March 2020.

[19] Rogers LC, Lavery LA, Joseph WS, Armstrong DG. All feet on deck-the role of podiatry during the COVID-19 pandemic: preventing hospitalizations in an overburdened healthcare system, reducing amputation and death in people with diabetes. J Am Podiatr Med Assoc. In Press. doi:10.7547/20-05.

[20] NICE guideline [NG160]. COVID-19 rapid guideline: dialysis service delivery. https://www.nice.org.uk/guidance/ ng160. Accessed at March 2020.

[21] American College of Surgeons. COVID-19 guidelines for triage of vascular surgery patients. https://www.facs.org/ covid-19/clinical-guidance/elective-case/vascular-surgery. Accessed 24 March 2020. 